\title{
A study on various pathogens causing corneal ulcers and their susceptibility to antibiotics
}

\author{
${ }^{1}$ A.V.Subba Rao, ${ }^{2}$ CH.S.B. Sailaja, ${ }^{3}$ Harshal Mendhe, ${ }^{4}$ Hanumanth N \\ I'Associate Professor, Dept. of Ophthalmology, Dr PSIMS \& RF, India) \\ ${ }^{2}$ (Fellow In Anterior Segment, Sankar Eye Foundation, India ) \\ ${ }^{3}$ (Assistant Professor, Dept. of Community Medicine, Dr PSIMS \& RF, India) \\ ${ }^{3}$ (Statistician, Dept. of Community Medicine, Dr PSIMS \& RF, India)
}

\begin{abstract}
:
Introduction: Corneal ulcer is one of the most common causes of preventable blindness especially in developing countries like India. Cataract and corneal diseases are major causes of blindness in countries with lessdeveloped economies. According to the World Health Organization, corneal diseases are among the major causes of vision loss and blindness in the world today, after cataract and glaucoma. As the corneal ulcer is the leading cause of this problem this study has been taken up to go into the details of etiology and incidence of various organisms causing corneal ulcers.

Methodology: This was a longitudinal study conducted at Ophthalmology OPD, Dr PSIMS \& RF, Gannavaram. 100 Patients having corneal ulcer were included as study participants. Most of the cases admitted were between age groups $11-80$. Children below the 10 years age group were excluded from the study.

Results: It was found that bacterial ulcers were common and accounted for 56\% of the cases. Fungal ulcers accounted for $20 \%$ of all the cases and NBG group were $14 \%$. Sterile cultures are due to the fact that the patient comes to the department after using antibiotics. Straphylococcus epdermidis was a commonest organism to cause an ulcer in compromised, malnourished and patient on steroids. The majority of fungal ulcers were due to direct injury to the eye with vegetable matter. Among the fungal ulcer, aspergillus fumigatus was commonest. The sensitivity found among isolated is highest with Gatifloxacin, Ciprofloxacin, Tobramycin and Gentamycin. Conclusion: Earlier the diagnosis and treatment based upon investigations, the cure rate of corneal ulcer are maximal.
\end{abstract}

Keywords: FB-Foreign Body, NBG-Non Bacterial Group, TKP-Total Kerato Plasty

\section{Introduction}

Corneal ulcer is one of the most common causes of preventable blindness especially in developing countries like India. Cataract and corneal diseases are major causes of blindness in countries with lessdeveloped economies. ${ }^{[1]}$ According to the World Health Organization, corneal diseases are among the major causes of vision loss and blindness in the world today, after cataract and glaucoma. ${ }^{[2]}$ As the corneal ulcer is the leading cause of this problem this study has been taken up to go into the details of etiology and incidence of various organisms causing corneal ulcers.

The majority of the cases are reported from rural areas who dependent upon agriculture. Malnourished and poorer income groins our country, trauma to cornea accounts for $60-80 \%$ of cases. Several studies have investigated the epidemiology of corneal ulceration, causative microorganisms, and effective treatments, particularly in the Indian subcontinent. However there is a paucity of information in the literature with regard to the experience in rural population of India ${ }^{[3,4,5,6]}$. Trauma may breakdown normal defense mechanism and allow resident flora of conjunctiva to colonize damaged corneal tissue or pathogenic organisms are inoculated into eye at time of injury. It has been one of the main ophthalmic problem as corneal ulceration can progress rapidly, threatening integrity of eye and producing significant tissue destruction with threat of vision loss and potential corneal perforation. Commonest predisposing factor for corneal ulcer observed was trauma or foreign body ${ }^{[7]}$ Present study was undertaken to know the common organisms causing corneal ulcers, incidence of various types of organisms causing corneal ulcers based on clinical and microbiological evaluation. Clinical and microbiological evaluation of corneal ulcers is of great help for control of ocular morbidity and blindness.

\section{Material and Methods}

This was a longitudinal study conducted at Ophthalmology OPD, Dr PSIMS \& RF, Gannavaram. 100 Patients having corneal ulcer were included as study participants. Most of the cases admitted were between age groups $11-80$. Children below the 10 years age group were excluded from the study. The scheme of work done in this study was the following.

a. History taking. 
b. Examination of cases

c. Investigation

d. Treatment

a. History taking: Age, sex, occupation, economic status and nourishment were recorded. The mode of onset was recorded and factors like injury or FB damaging the cornea were recorded. Previous ocular disease predisposing to ulcer was recorded. Systemic diseases like diabetics, Hypertension and HIV enquired. Exposure to chemicals and UV light rays enquired.

b. Examination of Cases: systemic and local examination was done and any associated eye conditions which may have predisposed to hypopyon were noticed visual acuity to be recorded in each case. The routine investigations like examination of urine and testing patency of lacrimal passage were done in all these cases.

c. Investigations: Conjunctival smears were examined for gram stain and $\mathrm{KOH}$ preparation. Corneal scraping of the base and margins after washing the conjunctival sac with $4 \%$ xylocaine. . The edges and base of the ulcer is scraped with a surgical blade no:15.smear for gram stain, geimsa stain and $\mathrm{KOH}$ preparation is done. The scraping is inoculated into blood agar, chocolate agar, nutrient agar broth and sabourods media. WBC and RBC count were done and are noted.

d. Treatment: Till the culture and sensitivity of corneal scarping is awaited fortified antibiotics are given. After the culture and sensitivity reports have arrived change in antibiotic is done as per the culture report.

\section{Results and Discussion}

A total of 100 corneal ulcer patients were included in the study. Corneal ulcer was more common in males $(56 \%)$ than in females (44\%) which explained by the fact that they were exposed to greater risk of injury to the eye. But a good number of females also suffered from corneal ulcer because they come from poorer classes and they have to work outside in the fields to earn their livelihood. $68 \%$ cases were from rural background and 32\% were urban background. The rural population has a light incidence of corneal ulcers of the frequent exposure to dust, dirt and foreign bodies also due to lack of proper education regarding eye diseases and also due to false belief and customs in treating the eye problems like application of natural irritants. $40 \%$ cases were agriculture labourer, $27 \%$ were industrial workers, $10 \%$ were house wives, $5 \%$ were employees. Agriculture labourer and industrial workers were more prone to injury and the foreign body in the eye. Hence occupation was an important factor in the causation of corneal ulcer. 34 cases had trauma as a predisposing cause, 24 cases had foreign body, 20 cases no cause, 20 cases had previous ocular disease and 2 cases had contact lenses. The age groups between 41-60 were more prone for corneal ulcers and this accounted for 74\% of cases. This is because of the working group falls in this category. The children below the age of 10 yrs were not included in this study. Corneal ulcers were more common among ill nourished and moderately nourished (90\%), malnutrition is a predisposing factor for corneal ulcer and delays healing. $2 \%$ cases belonged to rich economic status, $66 \%$ belong to poor economic status and $32 \%$ belonged to middle class.

Table-1: Organism isolated

\begin{tabular}{|l|l|l|}
\hline Organism & Number of cases & Percentage \\
\hline Bacteria & 56 & $56 \%$ \\
\hline Fungi & 20 & $20 \%$ \\
\hline NBG & 14 & $14 \%$ \\
\hline
\end{tabular}

It was found that bacterial ulcers were common and accounted for $56 \%$ of the cases. Fungal ulcers accounted for $20 \%$ of all the cases and NBG group were $14 \%$. Sterile cultures are due to the fact that the patient comes to the department after using antibiotics.

Table-2: Showing various Pathogenic Organisms Isolated in 100 cases of Clinically Diagnosed Corneal Ulcer.

\begin{tabular}{|l|l|l|}
\hline Type of organism & Number of cases & Percentage \\
\hline Staphylococcus epdermidis & 22 & $22 \%$ \\
\hline Pseudomonas aeruginosa & 10 & $10 \%$ \\
\hline Staphylococcus aureus & 10 & $10 \%$ \\
\hline Bacillus species & 6 & $6 \%$ \\
\hline Streptococcus pneumonia & 4 & $4 \%$ \\
\hline Klebsiella pneumonia & 2 & $2 \%$ \\
\hline E.coli & 2 & $2 \%$ \\
\hline $\begin{array}{l}\text { Fungal } \\
\text { Aspergillu fumigates }\end{array}$ & 14 & $14 \%$ \\
\hline Fusarium & 6 & $6 \%$ \\
\hline Viral & 10 & $10 \%$ \\
\hline
\end{tabular}




\begin{tabular}{|l|l|l|}
\hline NBG & 14 & $14 \%$ \\
\hline
\end{tabular}

Straphylococcus epdermidis was usually present in healthy conjuntival sac. It was a commonest organism to cause an ulcer in compromised, malnourished and patient on steroids. The majority of fungal ulcers were due to direct injury to the eye with vegetable matter. Among the fungal aspergillus fumigatus was commonest. We diagnosed viral keratitis based on patient history and clinical findings i.e., S/L examination and clinical features. One case of herpertic dendritic keratitis was reported which responded well to treatment. None of the patients examined wore any glasses to protect their eyes from injury and F.B. In all cases recorded that treatment was started with topical application fortified gentamycin and fortified ceftadizime hourly. The antibiotics were changed if necessary after receiving the culture report in each case. It was observed that frequent topical instillation of fortified antibiotic drops in the eye gave better results in many early and mild cases than with subconjunctival injections or systemic administration of antibiotics.

Table-3: Drug sensitivity of organisms isolated from bacterial corneal ulcer.

\begin{tabular}{|l|l|}
\hline Drug & Sensitivity positive \\
\hline Gatifloxacin & 28 \\
\hline Ciprofloxacin & 26 \\
\hline Tobramycin & 26 \\
\hline Ceftazidime & 18 \\
\hline Gentamycin & 20 \\
\hline Cloxacillin & 8 \\
\hline Chloramphenicol & 12 \\
\hline Ofloxacin & 8 \\
\hline
\end{tabular}

Table-4: Number of cases perforated and organism isolated.

\begin{tabular}{|l|l|}
\hline Organism & No. perforation \\
\hline Pseudomonas aeroginosa & 3 \\
\hline Staphylococcus epdermidis & 4 \\
\hline Bacillus & 1 \\
\hline Klebsiella & 1 \\
\hline
\end{tabular}

In this study it was found a total of cases were perforated out which two cases developed at anterior staphyloma and two cases developed adherent leucoma. For remaining five cases TKP done.

In the 3 month period 434 patients with central corneal ulceration were evaluated. A history of previous corneal injury was present in 284 patients (65.4\%). Cornea cultures were positive in 297 patients (68.4\%). Of those individuals with positive cultures $140(47.1 \%)$ had pure bacterial infections, $139(46.8 \%)$ had pure fungal infections, $15(5.1 \%)$ had mixed bacteria and fungi, and three (1.0\%) grew pure cultures of Acanthamoeba. The most common bacterial pathogen isolated was Streptococcus pneumoniae, representing $44.3 \%$ of all positive bacterial cultures, followed by Pseudomonas spp (14.4\%). The most common fungal pathogen isolated was Fusarium spp, representing 47.1\% of all positive fungal cultures, followed by Aspergillus spp (16.1\%). ${ }^{[8]}$ In the present study also it was found that bacterial ulcers were common and accounted for $56 \%$ of the cases. Fungal ulcers accounted for $20 \%$ of all the cases and NBG group were $14 \%$.

Out of 100 samples, bacteria were isolated from 47 samples. A total of 68 isolates of Staphylococcus aureus, Pseudomonas aeruginosa, and Klebsiella pneumoniae isolated from the samples. Among 68 isolates 31 were Staphylococcus aureus, 22 were Pseudomonas aeruginosa, 15 were Klebsiella pneumonia. The sensitivity and resistance pattern of Staphylococcus aureus, Pseudomonas aeruginosa, and Klebsiella pneumoniae against several antibiotics were observed by disc diffusion method on Mueller Hinton Agar (MHA- Hi-media) such as Moxifloxacin, Ofloxacin, Tobramycin, Cefazolin, Vancomycin, Chloramphenicol, Imipenem, Gentamicin, Ciprofloxacin, Ceftazidime. Staphylococcus aureus showed $22 \%$ to $78 \%$ resistance to these antibiotics. Pseudomonas aeruginosa showed $31 \%$ to $91 \%$ resistance to these antibiotics. Klebsiella pneumoniae showed $20 \%$ to $100 \%$ resistance to these antibiotics. The Moxifloxacin was $78 \%$ sensitive to S. aureus and Ciprofloxacin was $69 \%$ sensitive to P. aeruginosa and Ofloxacin was $80 \%$ sensitive to K. pneumoniae ${ }^{[9]}$ In the present study also $28 \%$ sensitivity was found in case of Gatifloxacin, in case of Ciprofloxacin $26 \%$ and in case of Ofloxacin it was $8 \%$.

\section{Conclusion}

- The incidence of corneal ulcer was more common in rural area $-68 \%$.

- Commonest predisposing factor observed was trauma or F.B.

- Most of the corneal ulcers seen in agricultural labour $-48 \%$ than the other occupation.

- Most of the fungal organisms were isolated from corneal ulcers seen in agricultural labour.

- Corneal ulcers most commonly seen in ill nourished and malnourished $90 \%$. 
- Most of the organisms isolated from corneal ulcer after lab investigations were bacteria $56 \%$.

- Among the bacteria most commonly seen organisms were staphylococcus epidermidis, pseudomonas aeroginosa, staphylococcus aereus.

- Fungi isolated from corneal ulcers - $20 \%$ among which most commonly isolated aspergillus species and fusarium.

- Viral corneal ulcer best diagnosed depending upon history and clinical picture $10 \%$.

- The sensitivity found among isolated is highest with Gatifloxacin, Ciprofloxacin, Tobramycin and Gentamycin.

- If the corneal becomes cloudy the only way to restore sight is to replace it with a healthy donar cornea through eye donation. Corneal transplantation (keratoplasty) in the most successful of all tissue transplants with overall success rate greater than $90 \%$. The success rate mostly depends on the cause of the corneal opacity.

- $\quad$ Earlier the diagnosis and treatment based upon investigations, the cure rate of corneal ulcer are maximal.

\section{References}

[1]. Garg P, Krishna PV, Stratis AK, Gopinathan U. The value of corneal transplantation in reducing blindness. Eye (Lond)2005;19:1106-14

[2]. Causes of blindness and visual impairment. Available from: http://www.who.int/blindness/causes/en/. [Last accessed on 2011 October 30]

[3]. Hagan M, Wright E, Newman M, et al. Causes of suppurative keratitis in Ghana. Br J Ophthalmol 1995;79:1024-8

[4]. Panda A, Sharma N, Das G, et al.Mycotic keratitis in children: epidemiologic and microbiologic evaluation. Cornea 1997;16:295-9.

[5]. Gugani HC, Talwar RS, Njoku-Obi AN, et al. Mycotic keratitis in Nigeria.A study of 21 cases. Br J Ophthalmol 1976;60:607-13.

[6]. Garg P, Gopinathan U, Choudhary K, et al. Keratomycosis: clinical and microbiologic experience with dematiaceous fungi. Ophthalmology 2000 107:574-80

[7]. Katara R, Patel N, Sinha M. A clinical microbiological study of corneal ulcer patient at western Gujarat, India. Acta Medica Iranica 2013; 51(6): 399-403

[8]. Srinivasan M, Gonzales CA, George C, Cevallos V, Mascarenhas JM, Asokan B et.al. Epidemiology and aetiological diagnosis of corneal ulceration in Madurai, south India. Br J Ophthalmol. 1997 Nov;81(11):965-71.

[9]. Jain Deepika, Musaddiq M. Combination therapy on pathogenic bacteria from corneal ulcers. International Journal of Applied Research 2015; 1(11): 878-881 